\title{
White Matter Integrity Changes and Neurocognitive Functioning in Adult- Late Onset DM1: A Follow-Up DTI Study
}

\author{
Garazi Labayru ( $\nabla$ garazi.labayru@ehu.eus ) \\ University of the Basque Country \\ Borja Camino-Pontes \\ BioCruces Health research Institute \\ Antonio Jimenez-Marin \\ BioCruces Health research Institute \\ Joana Garmendia \\ University of the Basque Country \\ Jorge Villanua \\ Osatek, Donostia University Hospital \\ Miren Zulaica \\ Biodonostia Health Research Institute \\ Jesus M Cortes \\ Ikerbasque \\ Adolfo López de Munain \\ Donostia University Hospital \\ Andone Sistiaga \\ University of the Basque Country
}

\section{Research Article}

Keywords: Myotonic Dystrophy Type 1, White Matter, DTI, neurodegeneration, neuropsychology

Posted Date: July 23rd, 2021

DOI: https://doi.org/10.21203/rs.3.rs-737338/v1

License: (c) (i) This work is licensed under a Creative Commons Attribution 4.0 International License. Read Full License

Version of Record: A version of this preprint was published at Scientific Reports on March 7th, 2022. See the published version at https://doi.org/10.1038/s41598-022-07820-1. 


\section{Abstract}

Background: Myotonic Dystrophy Type 1 (DM1) is a multisystemic disease that affects gray and white matter (WM) tissues. WM changes in DM1 include increased hyperintensities and altered tract integrity distributed in a widespread manner. However, the precise spatiotemporal changes are yet undetermined.

Methods: MRI data were acquired from 8 adult- and late-onset DM1 patients and 10 healthy controls (HC) at two different timepoints over 9.06 years. Fractional anisotropy (FA) variations were assessed with Tract-Based Spatial Statistics. Transversal and longitudinal intra- and intergroup analyses were conducted, along with correlation analyses with clinical and neuropsychological data.

Results: At baseline, reduced FA values were found in patients in the uncinate, anterior-thalamic, fronto-occipital, and longitudinal tracts. At follow-up, the WM disconnection was shown to have spread from the frontal part to the rest of the tracts in the brain. Furthermore, WM lesion burden was negatively correlated with FA values, while visuo-construction and intellectual functioning were positively correlated with global and regional FA values at follow-up.

Conclusion: DM1 patients showed a pronounced WM integrity loss over time compared to HC, with a neurodegeneration pattern that suggests a progressive anterior-posterior disconnection. The visuo-construction domain stands out as the most sensitive neuropsychological measure for WM microstructural impairment.

\section{Introduction}

Myotonic Dystrophy Type-1 (DM1) is an inherited autosomal dominant disorder, which affects multiple systems (e.g., muscular, cardiovascular, respiratory, gastrointestinal, endocrine), including the Central Nervous System (CNS). Although considered a rare disease, with an estimated worldwide prevalence of $0.5-18.1$ per 100,000 inhabitants ${ }^{1}$, DM1 is the most prevalent form of adult muscular dystrophy. Moreover, such prevalence is significantly higher in the geographical area of Gipuzkoa (Spain), which is considered the second most affected area in the world, with an estimated prevalence of 26.5 per 100,000 inhabitants $^{2}$.

Regarding CNS involvement, several symptoms such as excessive daytime sleepiness, fatigue, cognitive deficits, mood and behavior disorders, and certain personality traits, have been reported in varying degrees ${ }^{3}$. Additionally, neuroimaging studies have described brain abnormalities in both grey (GM) and whiter matter (WM) tissue.

According to a relatively recent review ${ }^{4}$, DM1 patients present general atrophy and GM volume reductions in all four cortical lobes (parietal, temporal, frontal and occipital), the basal ganglia, and the cerebellum. White matter lesions (WML) and white matter hyperintensities (WMH) have also been reported, as well as cortical volume loss and corpus callosum atrophy ${ }^{4,5}$. In addition, white matter (WM) tractography studies ${ }^{5,6}$ have shown significant diffusivity alterations, including an increase in mean diffusivity (MD), axial diffusivity (AD), radial diffusivity (RD), and a decrease in fractional anisotropy (FA). The multiple alterations found in WM have prompted some authors to state that DM1 is a predominantly white matter disease ${ }^{7}$.

Although still a matter of debate, there is a neurodegenerative hypothesis concerning the progression of the disease, supported by neuropathological, neuropsychological and neuroimaging evidence ${ }^{8-13}$. However, longitudinal studies assessing precise neurodegenerative patterns are scarce, and very few have assessed WM variations over time ${ }^{11,14-16}$. Of these studies, only two have attempted to analyze diffusivity values, yielding mixed results. Researchers agree that further investigation of WM pathology should be conducted with more valid and novel techniques such as Diffusion Tensor Imaging (DTI) tractography. This technique, along with a set of neuropsychological measures, are potentially critical for fully characterizing the status of individuals with $\mathrm{DM} 1^{17}$.

The aim of this study is to assess WM brain alterations by longitudinally analyzing diffusion images over a period of 10 years in clinical, neuropsychological and molecularly well characterized adult and late onset DM1 individuals. By comparing these individuals with healthy controls, it should be possible to delineate the pathway of disease progression and explore how these evolving patterns are associated with clinical and neuropsychological functioning.

\section{Results}

Across all participants, the mean time from baseline to follow-up was $9.06(S D=0.46)$ years, with no differences between groups $(D M 1: \bar{X}=9.24, S D=$ 0.33 ; $\mathrm{HC}: \bar{X}=8.91, \mathrm{SD}=0.5 ; t=-1.59, p=.131, d=.76)$. Regarding demographic characteristics of the sample, statistical analyses did not show significant differences between DM1 and HC groups in terms of gender, age, or years of education (Table 1).

Table 1. Demographic characteristics of the sample at baseline and follow-up 
DM1 $(\mathrm{N}=8)$

\begin{tabular}{lllllllll} 
& & Mean/N(\%) & (SD) & Mean/N(\%) & (SD) & Statistic & $\boldsymbol{p}$ & Effect size \\
\hline Gender & Male & $4(50 \%)$ & & $5(50 \%)$ & & $X^{2}=.000$ & 1 & $V=1$ \\
& Female & $4(50 \%)$ & & $5(50 \%)$ & & & & \\
\hline Age at baseline & & 46.5 & $(10.3)$ & 44.4 & $(8.6)$ & $t=-0.472$ & .643 & $d=.22$ \\
\hline Age at follow up & & 55.75 & $(10.25)$ & 53.3 & $(8.89)$ & $t=-0.543$ & .595 & $d=.26$ \\
\hline Years of education & 14.5 & $(7.35)$ & 14.13 & $(4.25)$ & $t=-0.125$ & .902 & $d=.06$
\end{tabular}

Note. DM1: Myotonic Dystrophy Type 1; HC: Healthy controls; SD: Standard Deviation. Descriptive data are shown as mean and SD for age at baseline, age at follow-up and years of education. Frequency $(\mathrm{N})$ and percentage (\%) are shown for gender, disease form, and inheritance.

From the eligible DM1 patients $(\mathrm{N}=22$, see Figure 1$)$, those who were finally included for analyses $(\mathrm{N}=8)$ did not differ from those who were excluded $(\mathrm{N}=14)$ in terms of age at baseline $(\mathrm{t}=1.07, \mathrm{p}=.296)$, muscular impairment at baseline $(\mathrm{U}=14.5, \mathrm{p}=.0 .86)$, years of education $(\mathrm{U}=36, \mathrm{p}=.167)$ and estimated intellectual functioning at baseline $(\mathrm{t}=-1.42, \mathrm{p}=.17)$. However, CTG expansion size at baseline was statistically larger in the excluded patients $(=820.29, \mathrm{SD}=510-27)$ than in the included patients $(=277.25, \mathrm{SD}=232.23): \mathrm{t}=2.82, \mathrm{p}=.01$.

WM lesion quantification revealed several differences between DM1 and HC both at baseline and follow-up (Table 2). In particular, DM1 patients showed significantly greater WM lesions cortically, but not in subcortical regions. Regions with larger WM lesion load at baseline were the total brain and temporal lobe; and at follow-up parieto-occipital and temporal lobes. Intragroup analyses of WM lesion load progression revealed a statistically significant increase in DM1 patients ( $z=-2.21 ; p=.027)$, but not in $\mathrm{HC}(z=-1.46 ; p=.142)$. Longitudinal analysis of clinical data revealed an absence of significant worsening of genetic defect $(\mathrm{t}=-2.109 ; p=.08)$ and muscular impairment $(\mathrm{Z}=-1 ; p=.317)$.

\section{Table 2. Clinical characterization of the sample at baseline and at follow-up}

BASELINE

\begin{tabular}{|c|c|c|c|c|c|c|c|c|c|c|c|c|c|c|}
\hline & $\mathrm{HC}$ & & DM1 & & & & & $\mathrm{HC}$ & & DM1 & & & & \\
\hline & Mean & SD & Mean & SD & $U$ & $p$ & $r$ & Mean & SD & Mean & SD & $U$ & $p$ & $r$ \\
\hline \multicolumn{15}{|l|}{$\begin{array}{l}\text { WM lesion } \\
\text { load }\end{array}$} \\
\hline GLOBAL & 1.1 & $(0,99)$ & 5.33 & (5.98) & 19.50 & .034 & 0.49 & 2.3 & $(2.83)$ & 6.89 & $(6.31)$ & 22.00 & .058 & 0.43 \\
\hline Frontal & 0.6 & $(0,7)$ & 1.89 & $(2.03)$ & 27.00 & .121 & 0.36 & 1.3 & $(1.34)$ & 2.44 & $(2.13)$ & 31.00 & .229 & 0.28 \\
\hline $\begin{array}{l}\text { Parieto- } \\
\text { occipital }\end{array}$ & 0.3 & $(0,48)$ & 1.89 & $(2.42)$ & 24.00 & .059 & 0.43 & 0.4 & $(0.7)$ & 2.67 & $(2.5)$ & 20.50 & .030 & 0.50 \\
\hline Temporal & 0 & $(0)$ & 1.22 & $(1.99)$ & 25.00 & .022 & 0.53 & 0 & $(0)$ & 1.56 & $(2.19)$ & 25.00 & .022 & 0.53 \\
\hline $\begin{array}{l}\text { Basal } \\
\text { ganglia }\end{array}$ & 0.1 & $(0,32)$ & 0.22 & $(0.67)$ & 44.00 & .878 & 0.04 & 0.5 & $(1.58)$ & 0.22 & $(0.67)$ & 45.00 & 1.000 & 0.00 \\
\hline Infratentorial & 0.1 & $(0,32)$ & 0.11 & $(0.33)$ & 44.50 & .939 & 0.02 & 0.1 & $(0.32)$ & 0 & $(0)$ & 40.50 & .343 & 0.22 \\
\hline CTG & & & 277.25 & $(232.23)$ & & & & & & 437.43 & $(385.21)$ & & & \\
\hline MIRS & & & 2.6 & $(0.89)$ & & & & & & 2.13 & $(1.25)$ & & & \\
\hline
\end{tabular}

Note. HC: Healthy Controls; DM1: Myotonic Dystrophy Type 1; SD: Standard Deviation. Descriptive data are shown as mean and SD for WM lesion load, CTG and MIRS. Frequency $(\mathrm{N})$ and percentage $(\%)$ are shown for disease form and inheritance.

The Supplementary material includes intragroup comparisons of DM1 patients' neuropsychological performance over time (Supplementary Table 1). Attention/processing speed was the only domain in which a statistically significant decrease was observed from baseline to follow-up (measured by standardized T values).

\section{Intergroup and intragroup tract integrity differences: transversal and longitudinal analyses}

Intergroup and intragroup differences in global and major tract integrity are shown in Figure 1. Global FA decreased significantly over time in both patients and HC, this decrease being more marked in DM1 patients. Intergroup differences were only significant at follow up.

All tracts analyzed showed significant intergroup differences in integrity, with the DM1 group showing the greatest WM disintegrity in all cases. Most of the differences were found at follow up. Regarding intragroup analysis, patients suffered a significant loss of integrity in the anterior thalamic radiation and superior longitudinal fasciculi; whereas $\mathrm{HC}$ showed a significant progressive loss in the inferior fronto-occipital fasciculus and superior 
longitudinal fasciculus. Group contrasts for all four possible combinations including transversal and longitudinal differences are shown in Supplementary Table 2, while the peak ROls of the studied WM tracts showing intergroup differences at baseline and follow-up can be found in Supplementary Tables 3 and 4, respectively.

\section{Association between tract integrity and clinical and neuropsychological data}

Correlation analyses between clinical (CTG, MIRS, WM lesion load) and neuropsychological performance and global FA at baseline did not reveal any significant correlations. At follow-up, global FA was only significantly associated with the visuo-construction domain $(r=0.79, p=.035)($ Figure 2$)$ and WM lesion load $(r=-0.76, p=.048)$ (Supplementary Table 5).

Regarding tract-based FA values, associations were only found at follow-up with neuropsychological performance (Figure 3 and Supplementary Table 5) and WM lesion load (Supplementary Table 5), except for an isolated negative correlation between WM lesion load and FA in the inferior longitudinal fasciculus $(r=-0.78, p=.037)$. In a similar manner, the visuo-construction domain was positively associated with regional FA values in the forceps major, forceps minor and inferior fronto-occipital fasciculus. Estimated IQ positively correlated with cingulum FA value. In all cases, better neuropsychological performance was associated with greater FA values. Greater WM lesion load correlated with lower FA values in the inferior fronto-occipital fasciculus, inferior longitudinal fasciculus and superior longitudinal fasciculus. No other significant associations were found between regional FA and other neuropsychological and clinical data.

\section{Discussion}

The present work constitutes the most extensive longitudinal DTI study in a sample of adult and late onset DM1 patients and HC to date. Over a period of nearly a decade, this study has revealed a progression in WM integrity impairment in adult and late onset DM1 population occurring as early as from the 40's to the 50's. The reduction in FA found at baseline in patients in comparison with HC was greater at follow-up, where WM integrity was compromised throughout all the tracts. This result points in the direction of the previously hypothesized neurodegenerative process and adds relevant information about the nature and early timing of this progression.

So far, only five MRI longitudinal studies including WM progression assessment ${ }^{11,14-16}$ have been published. Regarding WM lesion load, DM1 patients in this study - but not $\mathrm{HC}$ - showed a progression of WM lesion load over time, which supports the idea of a gradual deterioration, as suggested by some of the previous longitudinal studies ${ }^{14,15}$

Regarding WM integrity, a pattern of progressive degeneration was confirmed, evolving from the anterior thalamic radiation, the inferior frontooccipital, the longitudinal and the uncinate fasciculi, to a widespread involvement at follow-up. The damage shown at baseline indicates that patients suffer a WM alteration that disrupts multiple pathways, including the prefrontal-thalamic connection (anterior thalamic radiation), with decreases in volume observed for both areas in DM17,18-21; the connection between cortical lobes (inferior fronto-occipital and longitudinal fasciluli); and the connection between the limbic system and the frontal lobe (uncinate fasciculus). Regarding the latter, limbic system related difficulties such as emotional recognition have been described in DM1 population ${ }^{22-24}$ and have been found to be age-related ${ }^{25}$. The results reported in our study favor the idea that disrupted networks are potentially implicated in the various clinical symptoms manifested by the patients.

Regarding the progression of WM changes over time, a spatiotemporal pattern of anterio-posterior degeneration is suggested on the basis of our results. This anterio-posterior disconnection gradient has been reported with aging ${ }^{26-29}$, which reinforces the accelerated aging hypothesis in DM1. Notably, the progression reported here appears to start at a very young age (as early as in the 40s); an observation that is in accordance with the results reported in the most recently published work ${ }^{14}$, which found a reduced FA and increased MD in both cortical and subcortical regions in a group of patients below the age of 40 . However, evidence against this neurodegeneration process was reported by Gliem et al. (2019), who found a rather stable pattern of widespread microstructural WM alterations in middle-age patients. The disparities between these results could be partly explained by methodological differences, such as sample size, type of MRI scanner, or the timespan between assessments.

Some authors have suggested that the pattern of degradation in WM integrity could be a mediator of age-related changes in cognitive performance ${ }^{30}$. Neuropsychological performance (and more specifically visuo-construction abilities) was associated with greater WM impairment in this sample. Interestingly, this association was only found at follow-up, when patients differed more from HC in WM damage, suggesting that visuoconstruction abilities could be highly sensitive to changes in WM integrity. Both visuospatial and visuo-construction abilities have previously shown to be a hallmark of the DM1 cognitive profile ${ }^{16,31-33}$ and are reliable predictors of both cognitive and structural brain progressive degeneration ${ }^{11,14}$.

Clinical status has also found to be correlated with imaging changes in previous studies, with mixed results ${ }^{19-21,34-36}$. In the present work, disease severity, as measured by genetic and muscular defect, did not appear to be an indicator of WM impairment. It should be noted that most of the studies that found a correlation with clinical data employed heterogeneous samples including congenital and childhood forms. The present work was restricted to adult and late-onset patients, which could support the notion that, at least in these milder forms of the disease, WM damage might be inherent to the DM1 condition irrespective of disease severity. Nevertheless, we should bear in mind that CTG estimated in peripheral blood has been found to differ from that detected in other tissues (e.g., brain) ${ }^{37}$, which suggests that genotype-phenotype correlations should be interpreted with caution. 
The small sample size constitutes the major limitation of this study. The wide timespan in which the sample was assessed increased the rate of experimental deaths, which, along with the inherently higher mortality rate in the DM1 population, resulted in a reduced group of patients for subsequent analyses. For this reason, the results of this study should be interpreted with caution. Nonetheless, these findings indicate a potentially greater effect than that observed if the samples were larger. In the future, follow-up studies in other life stages and adding multiple timepoints (> 2) with shorter periods between assessments could help to monitor smaller and more specific time-dependent changes, and could also help to shed light on whether the natural history of brain changes follows a steady or irregular course. Moreover, future studies should make an effort to standardize MRI protocols, procedural issues, or sample characteristics (e.g., disease form, age span, or time from baseline to follow-up). This would help to provide reliable information on the natural progression of brain changes in DM1, which in turn, would enable the identification of potential biomarkers for the disease for future clinical trials.

Taken together, the current findings suggest that WM degeneration is of progressive nature in DM1, even at early life stages. However, this hypothesis can only be confirmed with further follow-up of the same cohorts. However, and precisely because of the young age of the sample, neurodevelopmental changes cannot be fully discarded. This debate can only be fully resolved by brain imaging studies, preferably those of a longitudinal nature that target patients as early as they are diagnosed.

\section{Conclusion}

Adult and late onset DM1 patients might suffer from a slow progressive neurodegenerative process of WM microstructural impairment at early ages, which appears to follow an anterior-posterior gradient and is associated with specific neuropsychological functions (i.e., visuo-construction). The need for further neuroimaging studies assessing patients from a longitudinal approach has been repeatedly highlighted in the literature. The hypothesized neurodegenerative nature of the disease has been supported by age-related changes observed in the brain structure of DM1 patients. However, the scarcity of studies means that the neurodevelopment-neurodegeneration debate still remains open.

\section{Methods}

\section{Participants}

The sample included in this study is part of a longitudinally followed-up cohort. From the 26 DM1 patients and 57 healthy controls (HC) assessed at baseline, 11 DM1 patients from the outpatient Neurology Service of the Donostia University Hospital and $11 \mathrm{HC}$ recruited from healthy volunteers and patients' relatives were re-scanned. The final sample included for the analyses was composed of 8 DM1 patients and $10 \mathrm{HC}$. A flow-chart showing the recruitment process and detailing the reasons for each excluded participant is shown in Figure 3 . From the healthy volunteers recruited at baseline, only those whose age, gender and years of education were equal or closely similar ( \pm 5 years at most) to any re-scanned patient were invited to participate in order to form demographically equivalent groups. The DM1 sample was distributed as follows regarding disease onset and inheritance pattern: 4 adult onset (age of onset: 20-40) (50\%) and 4 late onset patients (50\%) (age of onset >40); 3 patients with maternal inheritance (37.5\%), 4 with paternal inheritance (50\%) and 1 inherited from both parents $(12.5 \%)$.

Patients were excluded from the study if they had congenital (onset at birth), childhood (age of onset: 1-10) or juvenile (age of onset: 10-20) forms, history of major psychiatric or somatic disorder, acquired brain damage or alcohol or drug abuse, presence of corporal paramagnetic body devices that could impede an MRI study, and the presence of cerebral anomalies that could affect the MRI analysis. HC participants were required to satisfy the same inclusion criteria, except for the clinical diagnosis. These criteria were applied both at baseline and at follow-up.

This study was reviewed and approved by the Clinical Research Ethics Committee of the Gipuzkoa Health Area (DMRM-2017-01), in accordance with the principles of the Declaration of Helsinki and informed consent was obtained from all participants.

\section{Clinical and neuropsychological assessment}

Clinical and neuropsychological data were obtained at both baseline and follow-up by the research team's responsible neurologist and neuropsychologist, respectively.

Regarding clinical data, muscular impairment was assessed with the Muscular Impairment Rating Scale (MIRS) ${ }^{38}$, and CTG expansion size was determined from medical registries. Genetic assessment - PCR in DMPK alleles up to approximately 100 CTG and Southern blot analysis for larger expansions - was conducted in patients with no recent data available (within the last 5 years). The remaining clinical data concerning clinical form and maternal or paternal inheritance pattern were obtained from the patients' medical records.

For all DM1 patients, neuropsychological assessment was conducted by an experienced neuropsychologist in the hospital facilities. The examiner was blind to the clinical condition (i.e., disease form, CTG repeats, MIRS or inheritance pattern) and to the MRI results. The administered battery of neuropsychological tests included several tests and subtests, and standardized T values were obtained according to Spanish population-based normative data. The employed assessment tools were: Wechsler Adult Intelligence Scale - Third Edition (WAIS III) ${ }^{39}$, Rey-Osterrieth Complex Figure 
test (ROCF) ${ }^{40}$, Rey Auditory Verbal Learning Test (RAVLT) ${ }^{41}$, Stroop color and word test ${ }^{42}$, Raven's Progressive Matrices ${ }^{43}$, verbal fluency (semantic and phonemic) ${ }^{44,44}$ and California Computerized Assessment Package (CALCAP) ${ }^{45}$.

The converted scores were employed to calculate six cognitive-domains: visuo-construction (Block design from WAIS-III and ROCF copy), verbal memory (RAVLT immediate recall, RAVLT delayed recall, Total RAVLT), attention/ processing speed (Digit span from WAIS III, STROOP word, STROOP color and the following CALCAP subtests: Simple Reaction Time (RT), election RT, Sequential 1 RT, Sequential 2 RT), executive functioning (Total RAVEN, semantic fluency, phonemic fluency, STROOP color-word, STROOP interference) and visual memory (ROCF delayed recall), and intellectual functioning. The intellectual functioning domain was estimated from vocabulary and block design subtests of the WAIS III based on Sattler and Ryan (reliability rxx=.93; validity $r=.87$ ).

\section{MRI acquisition and data preprocessing}

Diffusion images were acquired using a 1.5 Tesla scanner (Achieva Nova, Philips) with a single-shot echo-planar diffusion imaging sequence (IVIMEPI) with the following parameters: voxel size $=1.75 \times 1.75 \times 2 \mathrm{~mm} 3 ; 60$ axial slices; TR =9,967 ms; TE = $66 \mathrm{~ms} ; \mathrm{matrix}$ size $=128 \times 128$. A diffusion gradient was applied across 32 non-collinear directions with b-value $=800 \mathrm{~s} / \mathrm{mm} 2$. Additionally, one set of images was acquired without diffusion weighting ( $b=0 \mathrm{~s} / \mathrm{mm} 2)$. All participants' MRI scans were acquired on the same scanner and with the same protocol at both timepoints.

White matter tract alterations were assessed with FSL v6.0.4 and Tract-Based Spatial Statistics (TBSS) (Smith et al., 2006) using Fractional Anisotropy (FA) images. First, all individual FA images were normalized non-linearly to the HCP1021-2mm template. Next, the mean image was computed across all participants and skeletonized to obtain the mean FA skeleton, which represents regions with high confidence bundles common to all participants and removing some of the subject-specific tract-based heterogeneities. FA images for each subject were then projected onto the mean skeletons.

WM lesion load was assessed according to the Wahlund scale (ARWM) ${ }^{47}$. When lesions $>5 \mathrm{~mm}$ were identified, severity was rated from 0 (no lesions) to 3 (diffuse involvement). Lesion location was quantified separately in five different regions: (1) the frontal area; (2) the parieto-occipital area; (3) the temporal area; (4) the infratentorial area, including the brain stem and cerebellum; and (5) the basal ganglia, including the striatum, globus pallidus, thalamus, internal and external capsules, and insula.

\section{Statistical analysis}

The SPSS (IBM SPSS Statistics 24) statistical package was used for sample description. Data were analyzed through inter-group comparisons to compare DM1 patients and HC, using contingency analysis (Chi-square) for categorical data and parametric (t-test) or non-parametric (MannWhitney U) for interval data, when appropriate. Intra-group analysis of the longitudinal evolution of clinical and neuropsychological data was conducted using the Wilcoxon signed-rank test or dependent t-test, when appropriate. In order to control for selective attrition bias, intra-group differences of eligible DM1 patients who were lost to follow-up and those who were included for analyses were conducted through independent sample comparisons (t-test or Mann Whitney $\mathrm{U}$, when appropriate).

For the main objectives of the present work, two analyses were conducted.

\section{Intergroup and intragroup tract integrity differences: transversal and longitudinal analyses}

For diffusion parameters, group comparisons at baseline and at follow-up were conducted using the randomise tool included in FSL, a nonparametric permutation test for finding cluster-based significant statistical differences between groups at the voxel level. Global FA values were obtained for each group at both timepoints. Major tracts over which alterations were assessed are shown in Supplementary Figure 1.

For multiple comparison correction, Threshold-Free Cluster Enhancement (TFCE) (Smith \& Nichols, 2009) was used, with 5000 iterations and Family Wise Error (FWE) corrected at $p=0.05$, thus ensuring that the chance of false positives was no more than $5 \%$, or equivalently, ensuring $95 \%$ confidence of no false positives. Group comparisons were conducted using two different contrasts: patient > control, and control > patients.

Global FA (the mean value across all voxels in the brain) and mean values of FA per tract were compared between groups and across longitudinal measures. First, a Kruskal-Wallis test was conducted between all the four possible values (HC baseline, DM1 baseline, HC follow-up, and DM1 followup), followed by additional Kruskal-Wallis tests as a post-hoc analysis for the following four comparisons: $\mathrm{HC}$ baseline vs $\mathrm{HC}$ follow-up, $\mathrm{HC}$ baseline vs DM1 baseline, DM1 baseline vs DM1 follow-up, HC follow-up vs DM1 follow-up). For multiple comparisons across the number of possible tracts, False Discovery Rate (FDR) was applied.

\section{Association between tract integrity and clinical and neuropsychological data}

For assessing the association between skeletonized FA images and both clinical and neuropsychological data, we only considered the intensity values belonging to the significant regions resulting from the group comparison at follow-up. These associations were analyzed at both baseline and at follow-up. For each subject, the mean value of FA within each significant tract was obtained and correlated with CTG expansion size, MIRS score, WM lesion load and the neuropsychological scores using the linear Pearson partial correlation index, controlling for the covariable of age. 
In all statistical analyses, missing values were handled by listwise deletion.

\section{Declarations}

\section{Data availability}

The data supporting the findings of this study and analysis code are available from the corresponding author on request.

\section{Acknowledgments}

The present study has been supported by funding from the Institute of Health Carlos III co-founded by Fondo Europeo de Desarrollo Regional-FEDER [grant numbers PI17/01231 and PI17/01841], CIBERNED (grant number: 609), the Basque Government [SAIO08-PE08BF01] and the University of the Basque Country (Neurosciences group: GIU20-057). BC was supported by a predoctoral grant from the Basque Government [PRE-2020-1-0187]. AJM was supported by a predoctoral grant from the Basque Government [PRE-2019-1-0070]. JG was supported by a predoctoral grant from the University of the Basque Country [PIF20/238].

\section{Author contributions}

G.L. contributed to designing the work, conducting the acquisition, analysis, and interpretation of data and to the writing of the original draft. B.C.P. and A.J.M. contributed to analysis and data visualization, and the writing, reviewing, and editing of the manuscript. J.G. contributed to the writing, reviewing, and editing of the manuscript. J.V. contributed to the analysis and interpretation of data and the writing, reviewing, and editing of the manuscript. M.Z. contributed to the data acquisition and the writing-review and editing of the manuscript. J.M.C. contributed to the interpretation of data and the writing, reviewing, and editing of the manuscript. A.L.M. contributed to the conceptualization, supervision, resource-management, and the writing, reviewing, and editing of the manuscript. A.S. contributed to conceptualization and design of the work, project administration, supervision, resource-management, and the writing, reviewing, and editing of the manuscript.

\section{Competing interests statement}

I declare that the authors have no competing interests as defined by Nature Research, or other interests that might be perceived to influence the results and/or discussion reported in this paper.

\section{References}

1. Theadom, A. et al. Prevalence of Muscular Dystrophies: A Systematic Literature Review. Neuroepidemiology 43, 259-268 (2014).

2. López de Munain, A. et al. Prevalence of myotonic dystrophy in Guipuzcoa (Basque Country, Spain). Neurology 43, 1573-1573 (1993).

3. Thornton, C. A. Myotonic dystrophy. Neurologic Clinics vol. 32 705-719 (2014).

4. Okkersen, K. et al. Brain imaging in myotonic dystrophy type 1. Neurology 89, 960-969 (2017).

5. Cabada, T. et al. Brain Involvement in Myotonic Dystrophy Type 1: A Morphometric and Diffusion Tensor Imaging Study with Neuropsychological Correlation. Archives of Clinical Neuropsychology 32, 401-412 (2017).

6. Lopez-Titla, M. M. et al. Cognitive Decline and White Matter Integrity Degradation in Myotonic Dystrophy Type I. Journal of Neuroimaging (2020) doi:10.1111/jon.12786.

7. Minnerop, M. et al. The brain in myotonic dystrophy 1 and 2: Evidence for a predominant white matter disease. Brain 134, 3527-3543 (2011).

8. Caillet-Boudin, M.-L. et al. Brain pathology in myotonic dystrophy: when tauopathy meets spliceopathy and RNAopathy. Front. Mol. Neurosci. 6, (2014).

9. Fernandez-Gomez, F. et al. Myotonic Dystrophy: an RNA Toxic Gain of Function Tauopathy? in Tau Biology (eds. Takashima, A., Wolozin, B. \& Buee, L.) vol. 1184 207-216 (Springer Singapore, 2019).

10. Gallais, B., Gagnon, C., Mathieu, J. \& Richer, L. Cognitive decline over time in adults with myotonic dystrophy type 1: A 9-year longitudinal study. Neuromuscular Disorders 27, 61-72 (2017).

11. Labayru, G. et al. Neurodegeneration trajectory in pediatric and adult/late DM1: A follow-up MRI study across a decade. Annals of Clinical and Translational Neurology 7, 1802-1815 (2020).

12. Modoni, A. et al. Cognitive impairment in myotonic dystrophy type 1 (DM1): A longitudinal follow-up study. J Neurol 255, 1737-1742 (2008).

13. Sansone, V. et al. Cognitive impairment in adult myotonic dystrophies: a longitudinal study. Neurol Sci 28, 9-15 (2007).

14. Cabada, T. et al. Longitudinal study in patients with myotonic dystrophy type 1: correlation of brain MRI abnormalities with cognitive performances. Neuroradiology (2020) doi:10.1007/s00234-020-02611-9.

15. Conforti, R. et al. Brain MRI abnormalities in the adult form of myotonic dystrophy type 1: A longitudinal case series study. Neuroradiol $J$ 29, 3645 (2016).

16. Gliem, C. et al. Tracking the brain in myotonic dystrophies: A 5-year longitudinal follow-up study. (2019) doi:10.1371/journal.pone.0213381. 
17. Wozniak, J. R., Mueller, B. A., Lim, K. O., Hemmy, L. S. \& Day, J. W. Tractography reveals diffuse white matter abnormalities in Myotonic Dystrophy Type 1. Journal of the Neurological Sciences 341, 73-78 (2014).

18. Labayru, G. et al. Regional brain atrophy in gray and white matter is associated with cognitive impairment in Myotonic Dystrophy type 1 . Neurolmage: Clinical 24, 102078 (2019).

19. Schneider-Gold, C. et al. Cortical and Subcortical Grey and White Matter Atrophy in Myotonic Dystrophies Type 1 and 2 Is Associated with Cognitive Impairment, Depression and Daytime Sleepiness. PLoS ONE 10, e0130352 (2015).

20. Sugiyama, A. et al. Brain gray matter structural network in myotonic dystrophy type 1. PLoS ONE 12, e0187343 (2017).

21. Zanigni, S. et al. Relationship of white and gray matter abnormalities to clinical and genetic features in myotonic dystrophy type 1. Neurolmage: Clinical 11, 678-685 (2016).

22. Serra, L. et al. Abnormal Cortical Thickness Is Associated With Deficits in Social Cognition in Patients With Myotonic Dystrophy Type 1. Front. Neurol. 11, 113 (2020).

23. Takeda, A., Kobayakawa, M., Suzuki, A., Tsuruya, N. \& Kawamura, M. Lowered sensitivity to facial emotions in myotonic dystrophy type 1. Journal of the Neurological Sciences 280, 35-39 (2009).

24. Winblad, S., Hellström, P., Lindberg, S. H. \& Hansen, S. Facial emotion recognition in myotonic dystrophy type 1 correlates with CTG repeat expansion. Journal of Neurology, Neurosurgery \& Psychiatry 77, 219-223 (2006).

25. Labayru, G. et al. Social cognition in myotonic dystrophy type 1: Specific or secondary impairment? PLoS ONE 13, e0204227 (2018).

26. Bennett, I. J., Madden, D. J., Vaidya, C. J., Howard, D. V. \& Howard, J. H. Age-related differences in multiple measures of white matter integrity: A diffusion tensor imaging study of healthy aging. Hum. Brain Mapp. NA-NA (2009) doi:10.1002/hbm.20872.

27. Fan, Q. et al. Age-related alterations in axonal microstructure in the corpus callosum measured by high-gradient diffusion MRI. Neurolmage 191, 325-336 (2019).

28. Gong, N.-J., Wong, C.-S., Chan, C.-C., Leung, L.-M. \& Chu, Y.-C. Aging in deep gray matter and white matter revealed by diffusional kurtosis imaging. Neurobiology of Aging 35, 2203-2216 (2014).

29. Xie, S. et al. Subcortical White Matter Changes with Normal Aging Detected by Multi-Shot High Resolution Diffusion Tensor Imaging. PLoS ONE 11, e0157533 (2016).

30. Madden, D. J. et al. Diffusion tensor imaging of cerebral white matter integrity in cognitive aging. Biochimica et Biophysica Acta (BBA) Molecular Basis of Disease 1822, 386-400 (2012).

31. Labayru, G., Aliri, J., Zulaica, M., López de Munain, A. \& Sistiaga, A. Age-related cognitive decline in myotonic dystrophy type 1: An 11-year longitudinal follow-up study. J Neuropsycho/ 14, 121-134 (2020).

32. Okkersen, K. et al. The cognitive profile of myotonic dystrophy type 1: A systematic review and meta-analysis. Cortex 95 , 143-155 (2017).

33. Sistiaga, A. et al. Cognitive/personality pattern and triplet expansion size in adult myotonic dystrophy type 1 (DM1): CTG repeats, cognition and personality in DM1. Psychol. Med. 40, 487-495 (2010).

34. Caso, F. et al. Cognitive Impairment in Myotonic Dystrophy Type 1 Is Associated with White Matter Damage. PLoS ONE 9, e104697 (2014).

35. Koscik, T. R. et al. White matter microstructure relates to motor outcomes in myotonic dystrophy type 1 independently of disease duration and genetic burden. Sci Rep 11, 4886 (2021).

36. van Dorst, M. et al. Structural white matter networks in myotonic dystrophy type 1. Neurolmage: Clinica/ 21, 101615 (2019).

37. Martorell, L. et al. Progression of somatic CTG repeat length heterogeneity in the blood cells of myotonic dystrophy patients. Human Molecular Genetics 7, 307-312 (1998).

38. Mathieu, J., Boivin, H., Meunier, D., Gaudreault, M. \& Begin, P. Assessment of a disease-specific muscular impairment rating scale in myotonic dystrophy. Neurology 56, 336-340 (2001).

39. Wechsler, D. WAIS-III: Escala de Inteligencia de Wechsler para Adultos III. (TEA Ediciones, 1999).

40. Rey, A. Copy and memory reproduction test of complex geometric figures. (TEA Ediciones, 2009).

41. Lezak, M., Howieson, H. \& Loring, D. Neuropsychological assessment. (Oxford University Press, 2004).

42. Golden, C. J. STROOP. Color and word test. (TEA Ediciones, 2001).

43. Raven, J. C., Court, J. H. \& Raven, J. Raven: Standard progressive matrices. (TEA Ediciones, 2001).

44. Casals-Coll, M. et al. Estudios normativos españoles en población adulta joven (proyecto NEURONORMA jóvenes): normas para los test de fluencia verbal. Neurología 28, 33-40 (2013).

45. Miller, E. N. CalCAP: California Computerized Assessment Package. (Norland Software, 1990).

46. Smith, S. M. et al. Tract-based spatial statistics: Voxelwise analysis of multi-subject diffusion data. Neurolmage 31, 1487-1505 (2006).

47. Wahlund, L. O. et al. A New Rating Scale for Age-Related White Matter Changes Applicable to MRI and CT. Stroke 32, 1318-1322 (2001).

48. Smith, S. \& Nichols, T. Threshold-free cluster enhancement: Addressing problems of smoothing, threshold dependence and localisation in cluster inference. Neurolmage 44, 83-98 (2009). 
Figures

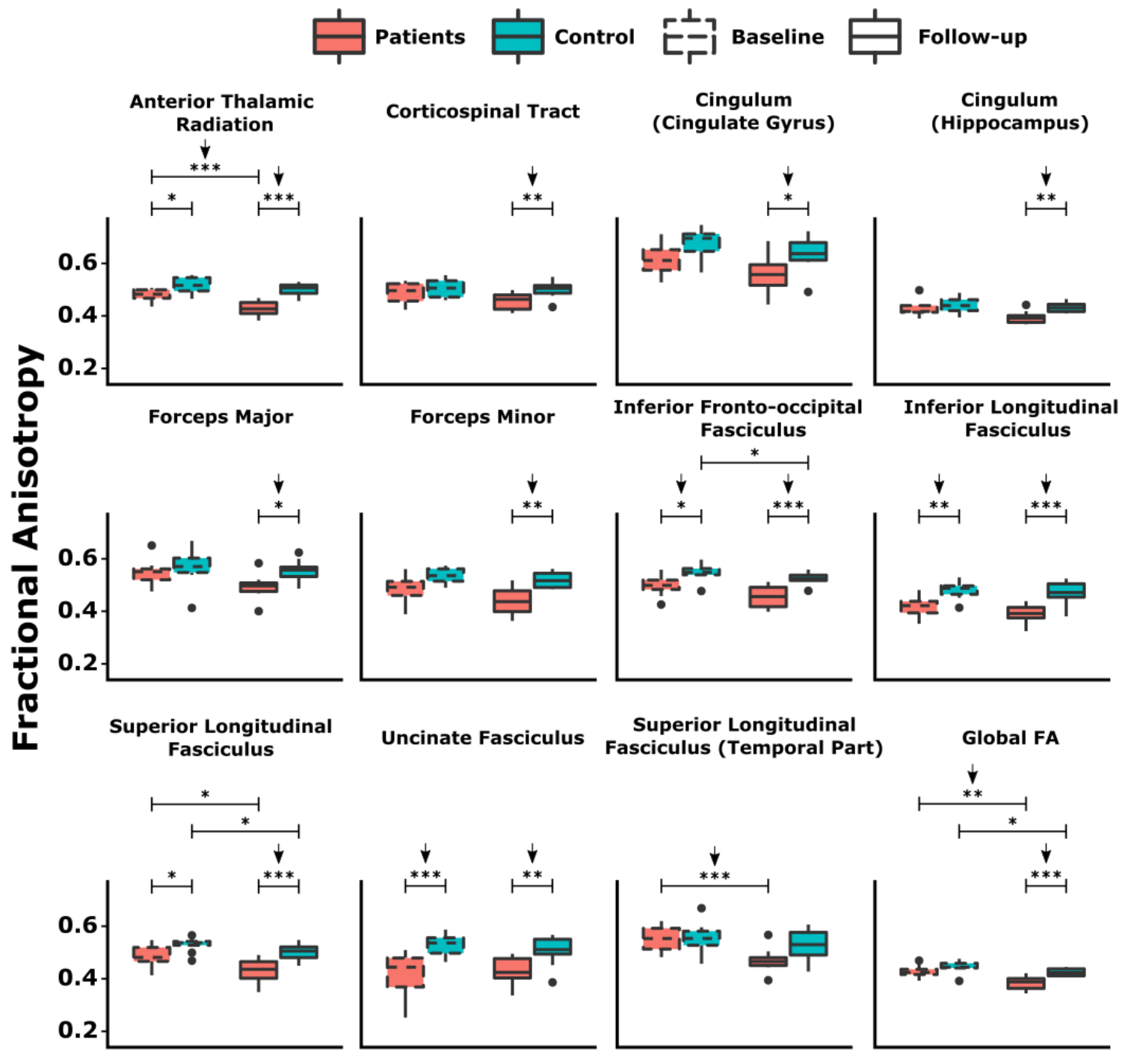

Figure 1

Differences in global and major tract integrity as measured by fractional anisotropy (FA) between groups and across longitudinal measures. For FA, lower values indicate reduced tract integrity. ${ }^{\star} p<0.05 ; * \star p<0.01 ; * \star \star p<0.005$. Arrows pointing down indicate comparisons surviving FDR multiple corrections. 
GLOBAL FA

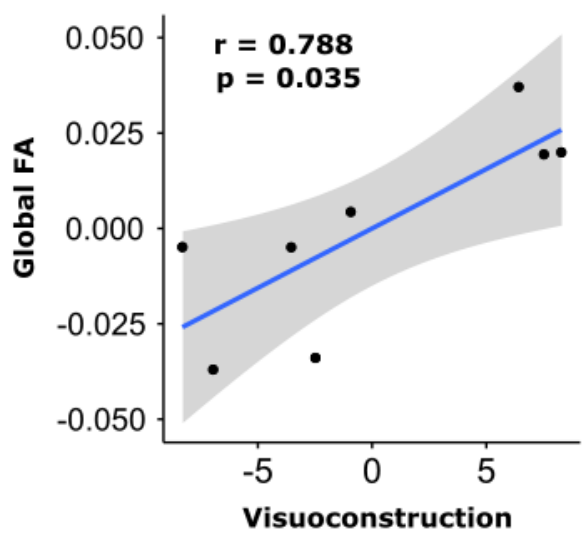

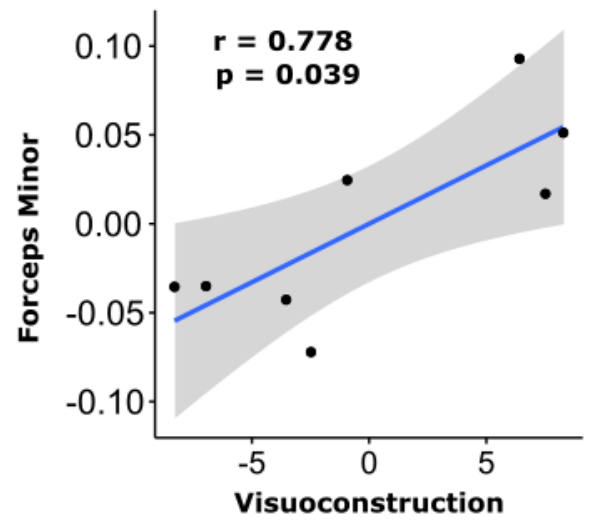

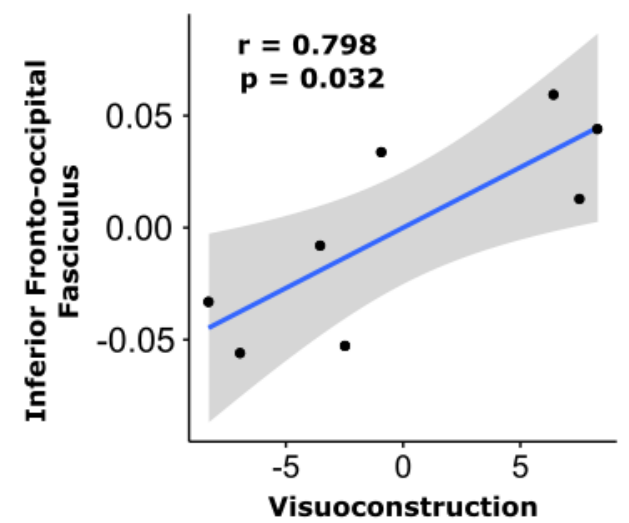

REGIONAL FA
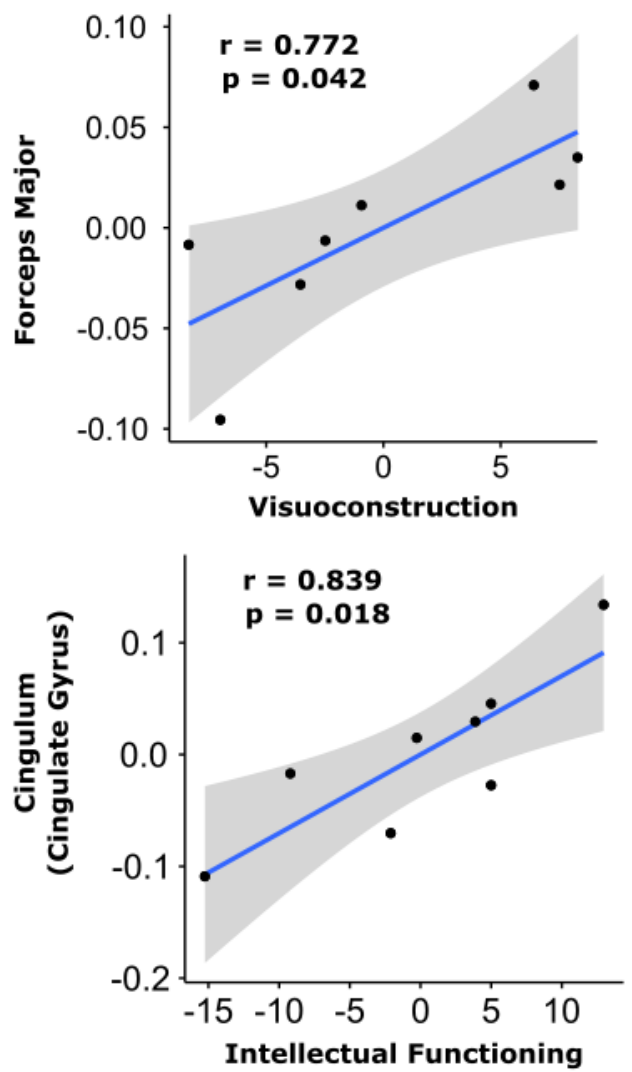

Figure 2

Association between tract integrity as measured by FA and neuropsychological scores. Shaded areas represent the $95 \%$ confidence interval in the regression model. Among all possible comparisons, only those plots with a statistically significant Pearson correlation $r$ (uncorrected, $p$-value $<0.05$ ) are displayed. 


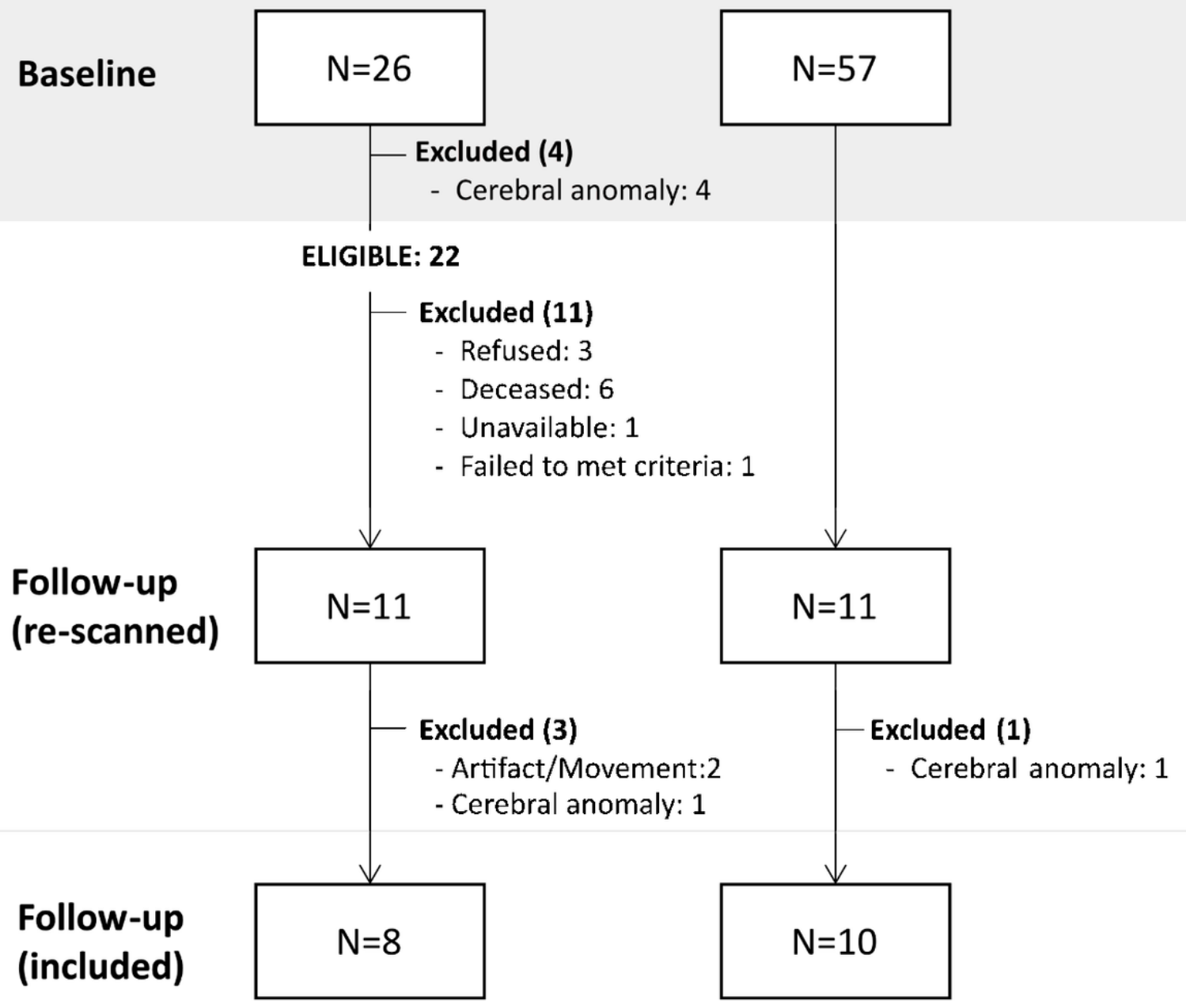

Figure 3

Flow-chart of the sample selection process

\section{Supplementary Files}

This is a list of supplementary files associated with this preprint. Click to download.

- Supplementarymaterial.docx 